\title{
The effect of Epstein-Barr virus infection in central nervous system on prognosis of patients with Epstein-Barr virus-associated hemophagocytic lymphohistiocytosis
}

\section{Zhili Jin}

Capital Medical University Affiliated Beijing Friendship Hospital

\section{Yini Wang}

Capital Medical University Affiliated Beijing Friendship Hospital

Lin Wu

Capital Medical University Affiliated Beijing Friendship Hospital

Zhao Wang ( $\sim$ wangzhao@ccmu.edu.cn )

Beijing Friendship Hospital, Capital Medical University

\section{Research article}

Keywords: Hemophagocytic lymphohistiocytosis , Central nervous system, Epstein-Barr virus infection , Allogeneic hematopoietic stem cell transplantation

Posted Date: October 14th, 2020

DOI: https://doi.org/10.21203/rs.3.rs-68405/v1

License: (9) This work is licensed under a Creative Commons Attribution 4.0 International License.

Read Full License 


\section{Abstract \\ Background}

This study was conducted to investigate the effect of Epstein-Barr virus (EBV) infection in the central nervous system(CNS) on the prognosis of Epstein-Barr virus-associated hemophagocytic lymphohistiocytosis (EBV-HLH) patients.

\section{Methods}

Clinical information of $88 \mathrm{EBV}$-HLH patients diagnosed and treated in our medical center from January 1 , 2017 to September 1, 2019 was included.

\section{Results}

The 88 patients were divided into 2 groups based on whether the result of their CSF EBV-DNA testing was positive. Statistical difference in survival time was found between the EBV + group and EBV- group(44 vs. $44 ; P=0.049)$. The 88 patients were separated into 2 groups according to whether underwent allogenic hematopoietic stem cell transplantation (allo-HSCT). There were statistically differences in the allo-HSCT group and non allo-HSCT group (60 vs. $28 ; P=0.040$ ). Among the $60 \mathrm{EBV}$-HLH patients who did undergo allo-HSCT, they were divided into 2 groups in the light of whether the result of CSF EBV-DNA testing was positive. Statistical difference in survival time was not found between the EBV + group and EBV - group( 32 vs. $28 ; P=0.540$ ). Among the $28 \mathrm{EBV}-\mathrm{HLH}$ patients who did not undergo allo-HSCT, they were divided into 2 groups based on whether the result of CSF EBV-DNA testing was positive. Significant statistical difference in survival time was found between the EBV + group and EBV - group(12 vs. $16 ; P=0.002)$. The 44 EBV-HLH patients who were EBV-DNA-positive in their CSF were separated into 2 groups for survival time analysis according to the highest copy number of EBV-DNA in the CSF, using $1.0 \mathrm{E}+04 \mathrm{copies} / \mathrm{ml}$ as the cutoff, and statistical difference in survival time was found between the two groups $(P=0.030)$.

\section{Conclusions}

EBV infection of the CNS was found to be a poor prognostic factor in EBV-HLH patients. The changes in EBV-DNA in CSF and the EBV-DNA copy number were found to affect the prognosis of EBV-HLH patients. Monitoring the changes in EBV-DNA copy number in the CSF was of great significance. Allo-HSCT improved the prognosis of EBV-HLH patients, including patients who tested positive for EBV-DNA in the CSF.

\section{Background}


$\mathrm{HLH}$ is a rapidly progressive and fatal disease characterized by high inflammatory cytokines production induced by undue immune activation. $\mathrm{HLH}$ can be separated into primary $\mathrm{HLH}$, which is induced by cell dysfunction due to gene mutation; and secondary HLH which is secondary to infection, malignant tumors, autoimmune diseases, and other causes [1]. Among the infection associated HLH, EBV-HLH is one of the most common and EBV-HLH patients suffer a much worse prognosis than other type of infection associated HLH[2]. A case of fatal EBV-HLH with severe involvement of the CNS showing florid hemophagocytosis in the choroid plexus, with extensive neuron loss and gliosis in the cerebrum, cerebellum, and brainstem has been reported[3].Currently, the incidence of CNS-HLH reported by different institutions is not completely consistent and varies between $10 \%$ and $73 \%[4,5]$. Neurological presentations are highly variable and include seizures, irritability, cranial nerve palsies, meningism, ataxia, signs of increased intracranial pressure, and altered consciousness $[4,5]$.There is a lot of attention on CSF abnormalities worldwide. It has been reported that the CSF abnormality rate in HLH patients was pretty high[6]. Hirst et al. reported that $76.47 \%$ of HLH patients had abnormal CSF[7]. More and more attention has been paid to HLH involving the CNS. However, existing reports mainly focused on the symptoms and imaging manifestations of the CNS, and etiological research involving the CSF has been especially rare. EBV plays an important role in the development and progression of HLH. This study was conducted to investigate the effect of EBV infection in the CNS on the prognosis of EBV-HLH patients.

\section{Methods}

\section{(1) Research Subject}

Clinical information of 88 EBV-HLH patients diagnosed and treated in our medical center from January 1 , 2017 to September 1, 2019 was included. All 88 patients had completed EBV-DNA testing in lumbar puncture and CSF whether they have CNS symptoms or not. The clinical information included patients' sex, age, EBV-DNA copy number in the CSF, the implementation of allo-HSCT, prognosis, and survival time. All patients met the following inclusion criteria.

1. met HLH-2004 diagnostic criteria [8];

2. high values (>500 copies/ml) for EBV-DNA copies in the peripheral blood mononuclear cells(PBMCs) or plasma (Pass the EBV international standard, namely: 09/260 (NIBSC number) for detection [9]);

3. primary HLH was excluded by HLH-related defective gene detection and whole genome sequencing if necessary, and lymphoma was excluded by repeated pathological biopsy of the focal area if there is an abnormal increase in FDG activity tested by positron emission tomographycomputed tomography (PET-CT), and bone marrow biopsy;

\section{(2) Detection of EBV-DNA in CSF}

Real time fluorescent quantitative PCR and TaqMan hydrolysis probe were used to amplify the viral characteristic gene sequences in the samples, and the standard curve was established for quantitative analysis. 


\section{(3) Survival Times}

Survival times of EBV-HLH patients were calculated from the date of diagnosis of EBV-HLH. All patients were followed up until death or 15 January 2020, whichever occurred first.

\section{(4) Treatment}

88 EBV-HLH patients were underwent the L-DEP regimen (PEG-aspargase ,doxorubicin, etoposide, methylprednisolone) [10] when they were hospitalized in our center (While they were applied HLH-94 regimen or HLH-2004 regimen in the other medical center, and the effect was not well) and allo-HSCT when they were relapse/refractory or central involvement.

\section{[5]Statistical Methods}

SPSS version 22.0 was used for statistical analysis. All the data that conformed to the normal distribution was represented by $\mathrm{x} \pm \mathrm{SD}$, while non-normal-distributed data were presented using the median and extreme values. Survival analysis (Kaplan - Meier analysis) was used to calculate the survival time of EBV-HLH patients. Statistical difference was defined as $\mathrm{P}<0.05$, significant statistical difference was defined as $\mathrm{P}<0.01$.

\section{Results}

(1) General conditions

Among the $88 \mathrm{EBV}$-HLH patients, 53 cases were male, 35 cases were female. The age of the patients ranged from 4 to 62 -year-old, and the median age was 26 -years. There were 33 patients $\leq 18$ years old and 55 patients $>18$ years old. Among the 33 patients who were $\leq 18$ years old, 17 cases $(17 / 33,51.5 \%)$ were positive for EBV-DNA in the CSF. Among the 55 patients who were $>18$ years old, 27 cases(27/55, 49.1\%) were positive for EBV-DNA in the CSF. Among the 88 patients, 60 patients underwent allo-HSCT and 28 patients did not.

In the 88 patients, 57 patients underwent only once EBV-DNA testing of the CSF via lumbar puncture, include 41 patients tested negative of their first CSF EBV-DNA testing and 16 patients tested positive. When the platelet count is $>50 \times 10^{9} / \mathrm{L}$, our center recommends regular follow-up EBV-DNA testing of the CSF collected through lumbar puncture, especially for patients whose CSF is positive for EBV-DNA. The patient is also given an intrathecal administration of $10 \mathrm{mg}$ methotrexate combined with $5 \mathrm{mg}$ dexamethasone for treatment during the follow-up lumbar puncture. Among the 16 positive cases mentioned above, 9 cases declined another lumbar puncture, and 7 cases were not able to undergo the lumbar puncture again because of a significant decrease in platelet count.

There were 31 patients with multiple lumbar punctures for EBV-DNA testing in the CSF, and 14 of them underwent 2 lumbar puncture tests; 5 of them underwent 3 lumbar puncture tests; 5 of them underwent 4 lumbar puncture tests; 3 of them underwent 5 lumbar puncture tests; 1 of them underwent 6 lumbar 
puncture tests; 2 of them underwent 7 lumbar puncture tests; 1 of them underwent 8 lumbar puncture tests.

(2) Survival analysis (Fig. 10)

The 88 patients were separated into 2 groups in the light of whether the result of CSF EBV-DNA testing was positive( $n=44$ positive cases, $n=44$ negative cases). Statistical difference in survival time was found between the two groups $(P=0.049)(F i g .1)$. The 88 patients were divided into 2 groups based on whether undergo allo-HSCT. Statistical difference in survival time was found between the two groups $(\mathrm{P}=$ 0.040)(Fig. 2). Among the $60 \mathrm{EBV}-\mathrm{HLH}$ patients who did undergo allo-HSCT, they were separated into 2 groups according to whether the result of their CSF EBV-DNA testing was positive $(n=32$ positive cases, $n$ $=28$ negative cases $)$. Statistical difference in survival time was not found between the two groups $(P=$ 0.540)(Fig. 3). Among the $28 \mathrm{EBV}$-HLH patients who did not undergo allo-HSCT, they were divided into 2 groups based on whether the result of their CSF EBV-DNA testing was positive $(n=12$ positive cases, $n=$ 16 negative cases). Significant statistical difference in survival time was found between the two groups $(P=0.002)$ (Fig. 4).

(3)Survival analysis ( The highest EBV-DNA copy number in CSF)

Among the 88 patients in this study, 44 patients were positive for EBV-DNA in the CSF. The highest EBVDNA copy number for the 44 patients with positive EBV-DNA tests of the CSF ranged between 3.7E + 02 copies $/ \mathrm{ml}$ and $2.8 \mathrm{E}+05$ copies $/ \mathrm{ml}$, and the median EBV-DNA copy was $1.0 \mathrm{E}+04 \mathrm{copies} / \mathrm{ml}(\mathrm{n}=21$ patients with EBV-DNA copy number $<1.0 \mathrm{E}+04$ copies $/ \mathrm{ml} ; \mathrm{n}=6$ patients with EBV-DNA copy number = $1.0 \mathrm{E}+04$ copies $/ \mathrm{ml} ; \mathrm{n}=17$ patients with EBV-DNA copy number $>1.0 \mathrm{E}+04 \mathrm{copies} / \mathrm{ml}$ ). The patients were divided into two groups for survival time analysis based on their highest EBV-DNA copy number with $1.0 \mathrm{E}+04 \mathrm{copies} / \mathrm{ml}$ as the cut-off $(\mathrm{n}=27$ patients with EBV-DNA copy number $\leq 1.0 \mathrm{E}+04 \mathrm{copies} / \mathrm{ml}$, and $\mathrm{n}=17$ patients with EBV-DNA copy number $>1.0 \mathrm{E}+04$ copies $/ \mathrm{ml}$ ). Statistical difference in survival time was found between the two groups(median overall survival 17.0 versus 5.2 months, respectively; $\mathrm{P}=$ 0.030)(Fig. 5).

(4)Survival analysis( The first CSF EBV-DNA testing)(Fig. 11)

The 88 patients were split into 2 groups according to whether the result of their first CSF EBV-DNA testing was positive( $n=43$ positive cases, $n=45$ negative cases). Statistical difference in survival time was found between the two groups $(P=0.035)$ (Fig. 6).

(5)Survival analysis (EBV-DNA testing results before allo-HSCT)

The 60 patients with allo-HSCT were divided into two groups for survival time analysis based on their results of EBV-DNA testing of the CSF before the transplantation $(n=26$ positive cases, $n=34$ negative cases).Statistical difference in survival time was not found between the two groups $(P=0.608)(F i g .7)$.

(4)Survival analysis(Last CSF EBV-DNA testing)

The $31 \mathrm{EBV}-\mathrm{HLH}$ patients with multiple lumbar puncture tests were divided into two groups in the light of whether the result of the last EBV-DNA test of their CSF was positive for analysis of survival time $(n=12$ positive cases, $n=19$ negative cases $)$, and there were statistically differences in the two groups $(P=$ 0.034)(Fig. 8).

(5)Survival analysis(Changes in EBV-DNA in CSF) 
The 27 patients who underwent multiple lumbar puncture tests and also had a positive result of the first EBV-DNA test of their CSF were divided into two groups based on whether the EBV-DNA tests of their CSF changed from positive to negative(There were 16 patients with their CSF EBV-DNA testing results changed from positive to negative, and 11 patients with their EBV-DNA testing results continued to be positive), and statistical difference in survival time was found between the two groups $(P=0.038)($ Fig. 9). Of the 16 patients whose CSF EBV-DNA testing results changed from positive to negative, all patients were treated with intrathecal injection of dexamethasone and methotrexate; 15 of them underwent alloHSCT, 1 patient did not receive allo-HSCT.

\section{Discussion}

$\mathrm{HLH}$ is a severe or even fatal multisystem disorder caused by a hereditary or acquired immunoregulatory abnormality. CNS involvement is a poor prognostic component, better neuroradiological surveillance may be beneficial for patient management and outcome[11]. A case of fatal EBV-HLH with severe involvement of the central nervous system showing florid hemophagocytosis in the choroid plexus, with extensive neuron loss and gliosis in the cerebrum, cerebellum, and brainstem has been reported[3].Between 1996 and 2007, nine children with HLH with CNS involvement were identified in a single centre.

Neuroradiological findings from CT, MRI, and proton MR spectroscopy, and the clinical records of the nine children were retrospectively reviewed[11]. A study aimed to retrospectively describe and illustrate the MRI appearances of CNS involvement by HLH in a cohort of seven Australian children from two paediatric centres between 2000 and 2011[12].A substantial proportion of HLH patients suffer neurological sequelae, and patients with abnormal CSF have increased risk of mortality and neurological sequelae[13].More and more attention has been paid to HLH involving the CNS. However, existing reports mainly focused on the symptoms and imaging manifestations of the CNS, and etiological research involving the CSF has been especially rare. EBV plays an important role in the development and progression of HLH. Studies from our center by Wang et al. [14] have shown that significantly longer survival $(p=0.018)$ was observed in patients whose EBV-DNA was reduced $>100$-fold compared with those EBV-DNA was reduced < 100-fold after treatment and patients with low EBV-DNA copy number had a longer survival time. This indicates the close relationship between EBV and the prognosis of HLH patients. This study was conducted to investigate the effect of EBV infection in the CNS on the prognosis of EBV-HLH patients.

The 88 patients were divided into 2 groups according to whether the CSF EBV-DNA tests were positive, and there were statistically differences in the two groups $(P=0.049)$. The 88 patients were separated into 2 groups based on whether the first CSF EBV-DNA testing was positive. Statistical difference was also found between the two groups $(P=0.035)$. These indicated that the CSF EBV-DNA testing results were associated with the prognosis of EBV-HLH patients.

As we all know, the only proven effective treatment for relapse/refractory or central involvement EBV-HLH patients is allo-HSCT. Therefore, we divided the 88 patients into 2 groups according to whether they had received allo-HSCT. Among the non allo-HSCT group, they were separated into 2 groups based on whether 
the result of CSF EBV-DNA testing was positive. Significant statistical difference was found between the two groups $(P=0.002)$. This also indicated that EBV infection in the CNS affected the prognosis and was a poor prognostic factor for patients with EBV-HLH. Among the allo-HSCT group, they were divided into 2 groups according to whether the result of their CSF EBV-DNA testing was positive. There were not statistically differences in the two groups $(P=0.540)$. At the same time, the 60 patients with allo-HSCT were separated into two groups based on their results of CSF EBV-DNA testing before the transplantation, and statistical difference also was not found between the two groups $(P=0.608)$. The 88 patients were divided into 2 groups according to whether undergo allo-HSCT. Statistical difference was found between the two groups $(P=0.040)$. These in turn indicated that allo-HSCT improved the prognosis of EBV-HLH patients, including EBV-HLH patients who had positive CSF EBV-DNA testing results.

The $31 \mathrm{EBV}-\mathrm{HLH}$ patients with multiple lumbar puncture tests were divided into two groups based on whether the result of the last CSF EBV-DNA test was positive and statistical difference in survival time was found between the two groups $(P=0.034)$. The 27 patients who underwent multiple lumbar puncture tests and also had a positive result of the first EBV-DNA test of their CSF were separated into two groups based on whether the CSF EBV-DNA tests changed from positive to negative, and statistical difference was found between the two groups $(P=0.038)$. The 44 EBV-HLH patients who were EBV-DNA-positive in their CSF were divided into two groups based on the highest copy number of EBV-DNA, and there were statistically differences between the two groups $(P=0.030)$. These findings also demonstrated that the changes in EBV-DNA in the CSF and the number of EBV-DNA copy numbers were associated with the prognosis of EBV-HLH patients, suggesting that it is particularly important to monitor the EBV-DNA copy number in the CSF of EBV-HLH patients who tested positive for EBV-DNA in their CSF.

Of the 16 patients whose CSF EBV-DNA testing results changed from positive to negative, all patients were treated with intrathecal injection of dexamethasone and methotrexate; 15 of them underwent alloHSCT, but the other 1 patient did not receive allo-HSCT, the EBV-DNA testing result of this patient changed from positive to negative after receiving the intrathecal injection of dexamethasone and methotrexate. Regarding the CNSHLH prognosis, current views generally consider that intrathecal injection does not have a significantly active function on the final outcome of CNSHLH and that more often alloHSCT is required.[15]. But there are also articles that think multiple rounds of repeated intrathecal injection therapy can improve the prognosis of CNSHLH patients who cannot receive alloHSCT [16]. The benefits of intrathecal injection of dexamethasone and methotrexate were undeniable in EBV-HLH patients.

\section{Conclusions}

EBV infection of the CNS was found to be a poor prognostic factor in EBV-HLH patients. The changes in EBV-DNA in CSF and the EBV-DNA copy number were found to affect the prognosis of EBV-HLH patients. Monitoring the changes in EBV-DNA copy number in the CSF was of great significance. Allo-HSCT improved the prognosis of EBV-HLH patients, including patients who tested positive for EBV-DNA in the CSF. Thus, these patients should receive allo-HSCT.

\section{Abbreviations}


$\mathrm{HLH}$

Hemophagocytic lymphohistiocytosis

CNS

Central nervous system

EBV

Epstein-Barr virus

EBV-HLH

EBV associated HLH

CSF

Cerebrospinal fluid

Allo-HSCT

Allogeneic hematopoietic stem cell transplantation

PBMCs

Peripheral blood mononuclear cells

\section{Declarations}

Ethics approval and consent to participate: All patients had signed the informed consent of lumbar puncture before lumbar puncture.

Consent for publication: Not applicable.

Availability of data and material: Please contact author for data requests.

Competing interests: The authors declare that they have no competing interests.

Funding: This work was supported by National Natural Science Foundation of China (No.81871633); Beijing Natural Science Foundation (No.7181003); Beijing Municipal Administration of Hospitals Clinical Medicine Development of Special Funding(ZYLX201702); Beijing Municipal Administration of Hospitals' Ascent Plan(DFL20180101); Beijing Municipal Administration of Hospitals Incubating Program(PX2018003); Beijing Municipal Administration of Hospitals Clinical Technology Innovation Project(XMLX201803). The funding bodies support the publication fee for us and have no role in the process of study design, data collection, analysis, and writing the manuscript.

Authors' contributions: ZJ collected and analyzed data, and drafted the manuscript.

YW, LW, ZW participated in the design of the study and performed the statistical analysis. All authors read and approved the final manuscript.

Acknowledgements: Not applicable.

\section{References}


[1] Tothova Z, Berliner N. Hemophagocytic Syndrome and Critical Illness: New Insights into Diagnosis and Management[J]. J Intensive Care Med, 2015, 30(7): 401-412.

[2]Imashuku S. Treatment of Epstein-Barr virus-related hemophagocytic lymphohistiocytosis (EBV-HLH); update 2010[J]. J Pediatr Hematol Oncol, 2011, 33:35-39.

[3]Magaki S, Ostrzega N, Ho E, et al.Hemophagocytic lymphohistiocytosis associated with Epstein-Barr virus in the central nervous system[J].Hum Pathol. 2017, 59:108-112.

[4]Jovanovic A, Kuzmanovic M, Kravljanac R,et al. Central nervous system involvement in hemophagocytic lymphohistiocytosis: A single-center experience[J]. Pediatr Neurol, 2014, 50:233-237.

[5]Weisfeld-Adams JD, Frank Y, Havalad V, et al. Diagnostic challenges in a child with familial hemophagocytic lymphohistiocytosis type $3(\mathrm{FHLH} 3)$ presenting with fulminant neurological disease[J]. Childs Nerv Syst ,2009, 25:153-159.

[6] Horne A, Trottestam H, Aricò $M$, et al. Frequency and spectrum of central nervous system involvement in 193 children with haemophagocytic lymphohistiocytosis[J]. Br J Haematol, 2008,140:327-335.

[7] Hirst WJ, Layton DM, Singh S, et al. Haemophagocytic lymphohistiocytosis: Experience at two U.K. centres[J]. Br J Haematol, 1994,88:731-9.

[8]Henter JI, Horne A, Aricó M, et al. HLH-2004:Diagnostic and therapeutic guidelines for hemophagocytic lymphohistiocytosis[J]. Pediatr Blood Cancer, 2007, 48:124-131.

[9]Fryer JF, Heath AB, Wilkinson DE, et al. A collaborative study to establish the 1st WHO International Standard for Epstein-Barr virus for nucleic acid amplification techniques[J].Biologicals,2016;44(5):423-33.

[10]Jingshi Wang, Yini Wang, Lin Wu, et al.PEG-aspargase and DEP regimen combination therapy for refractory Epstein-Barr virus-associated hemophagocytic lymphohistiocytosis[J].J Hematol Oncol.2016 9;9(1):84.

[11]Goo HW, Weon YC. A spectrum of neuroradiological findings in children with haemophagocytic lymphohistiocytosis[J]. Pediatr Radiol, 2007,37:1110-1117.

[12]Guandalini M, Butler A, Mandelstam S.Spectrum of imaging appearances in Australian children with central nervous system hemophagocytic lymphohistiocytosis[J]. J Clin Neurosci.2014,21(2):305-310.

[13] Horne A, Trottestam $\mathrm{H}$, Aricò $\mathrm{M}$, et al. Frequency and spectrum of central nervous system involvement in 193 children with haemophagocytic lymphohistiocytosis[J] . Br J Haematol.2008 ,140:327-335

[14]Wang J,Wang Y,Wang Z, et al. PEG-aspargase and DEP regimen combination therapy for refractory Epstein-Barr virus-associated hemophagocytic lymphohistiocytosis[J]. J Hematol Oncol, 2016, 9: 84. 
[15]Sparber-Sauer M, Hönig M, Schulz AS, et al. Patients with early relapse of primary hemophagocytic syndromes or with persistent CNS involvement may benefit from immediate hematopoietic stem cell transplantation[J]. Bone Marrow Transplant, 2009, 44:333-338.

[16]Yue Song, Rui-Jun Pei, Yi-Ni Wang, et al. Central Nervous System Involvement in Hemophagocytic Lymphohistiocytosis in Adults: A Retrospective Analysis of 96 Patients in a Single Center[J]. Chin Med J ,2018,131:776-783.

\section{Figures}

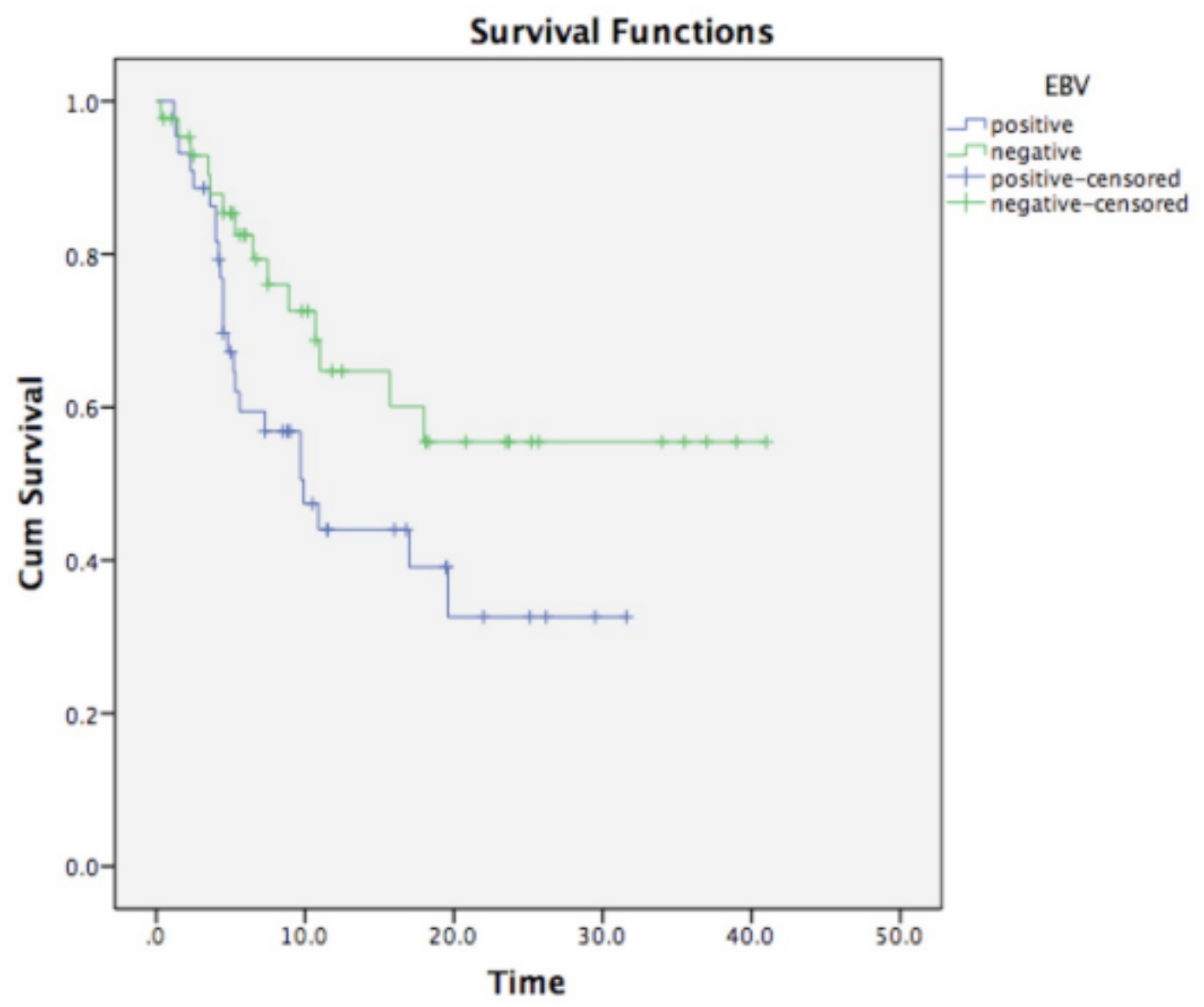

\section{Figure 1}

Survival curves of 2 groups whose CSF EBV-DNA testing was positive and negative. 


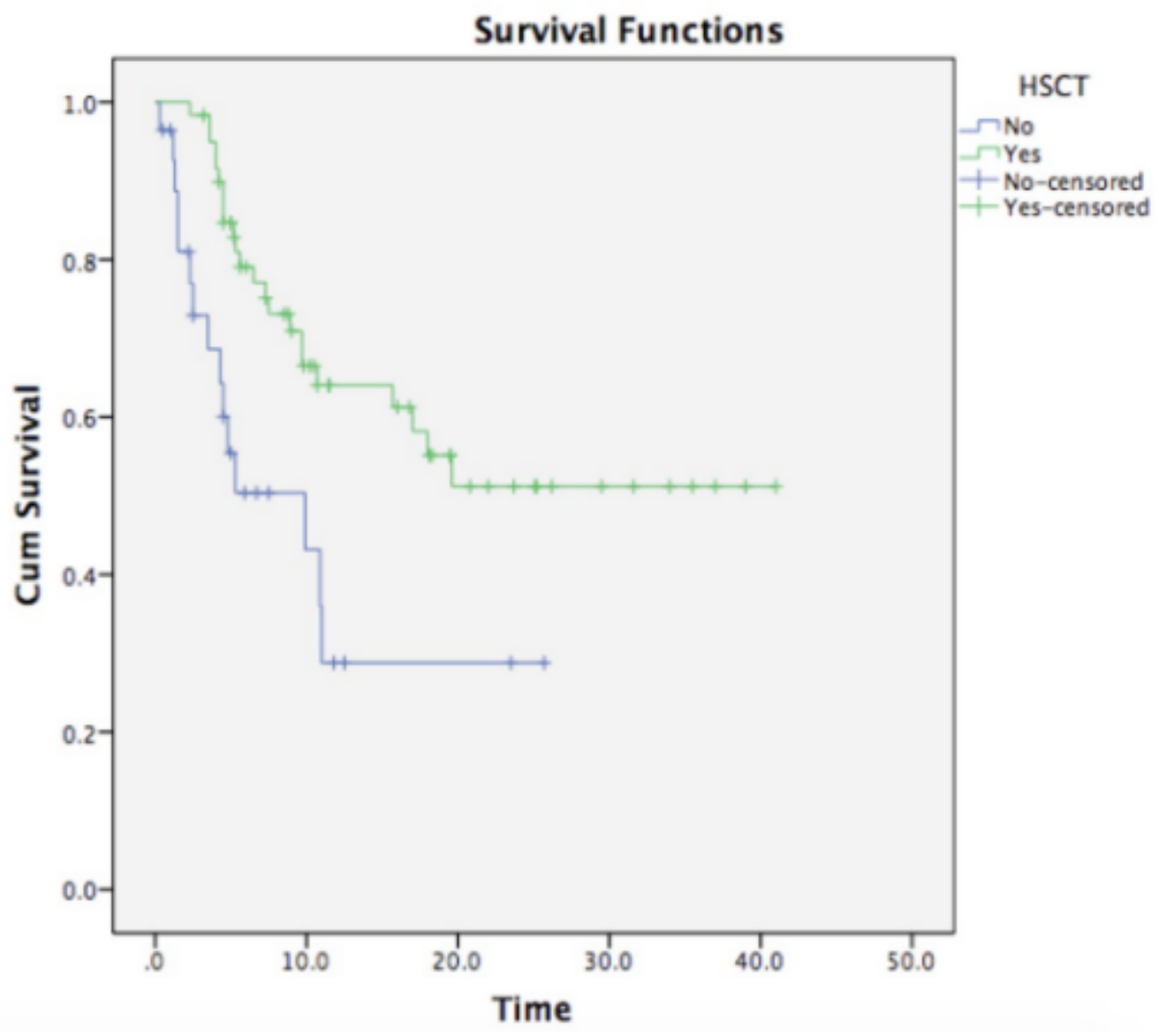

Figure 2

Survival curves of patients who did undergo allo-HSCT and did not undergo allo-HSCT.

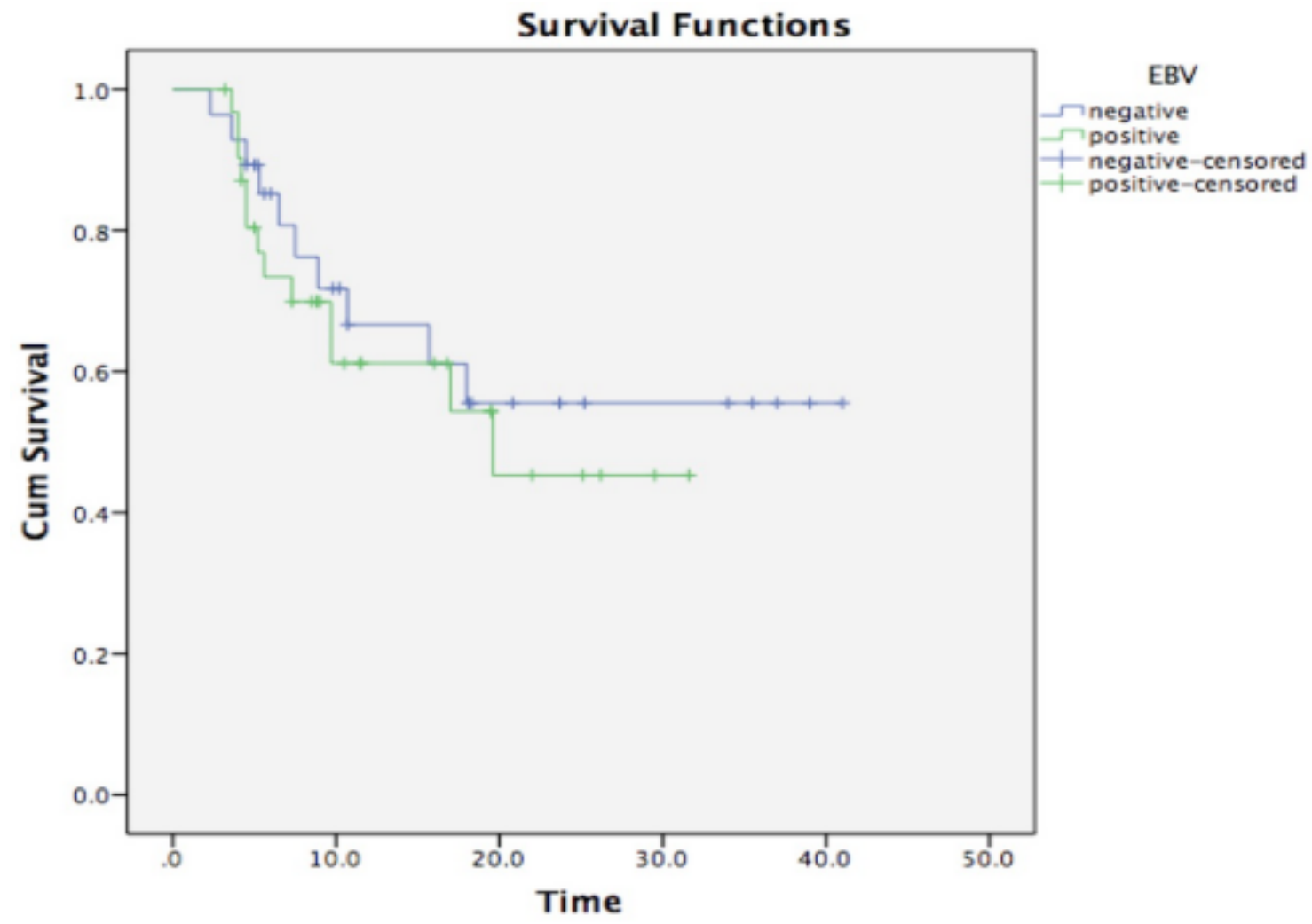

Page 11/19 


\section{Figure 3}

Survival curves of 2 groups whose CSF EBV-DNA testing was positive and negative in patients who did undergo allo-HSCT .

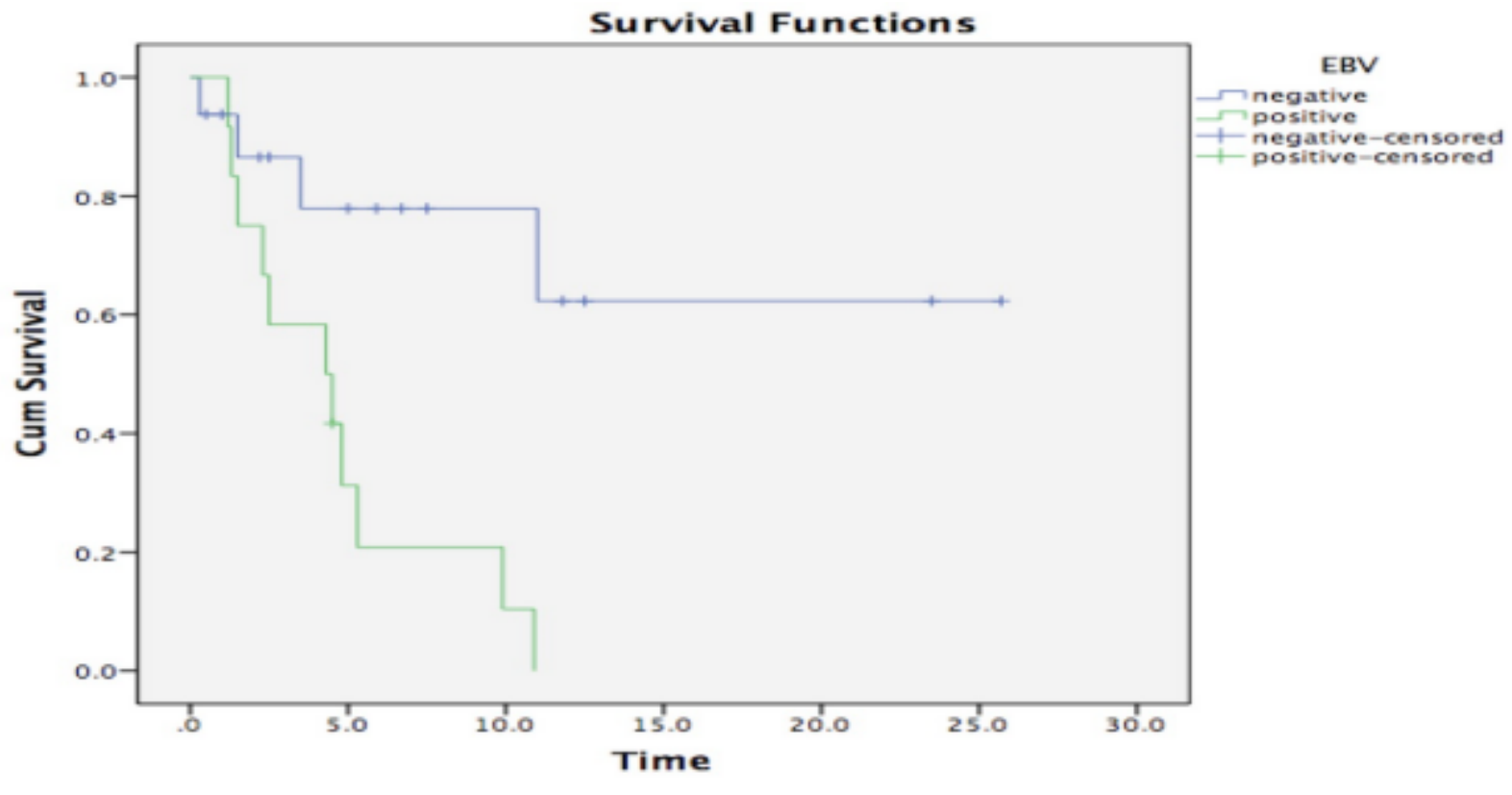

\section{Figure 4}

Survival curves of 2 groups whose CSF EBV-DNA testing was positive and negative in patients who did not undergo allo-HSCT. 


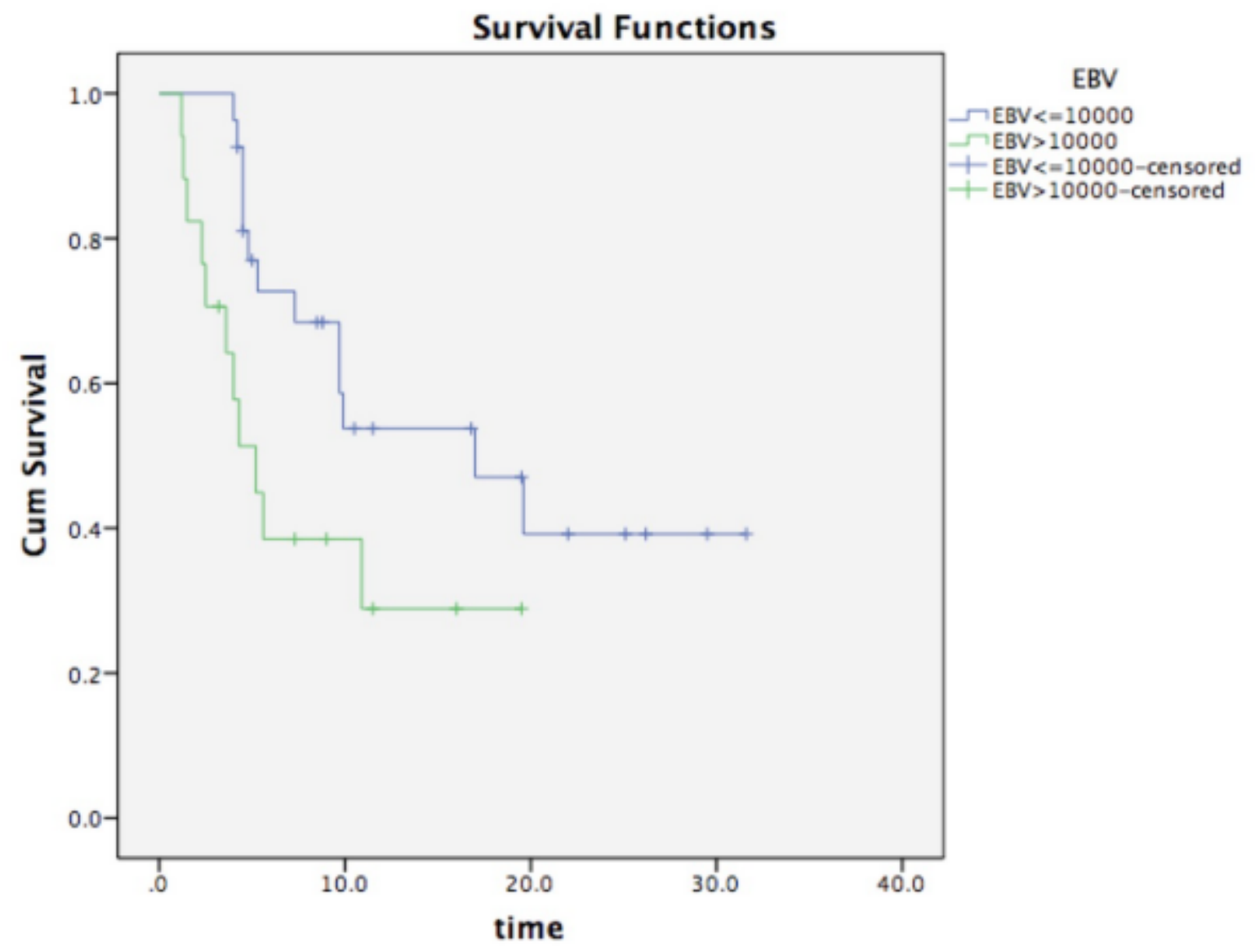

Figure 5

Survival curves of whose highest EBV-DNA copy number $\leq 1.0 \mathrm{E}+04$ copies $/ \mathrm{ml}$ and $>1.0 \mathrm{E}+04$ copies/ml. 


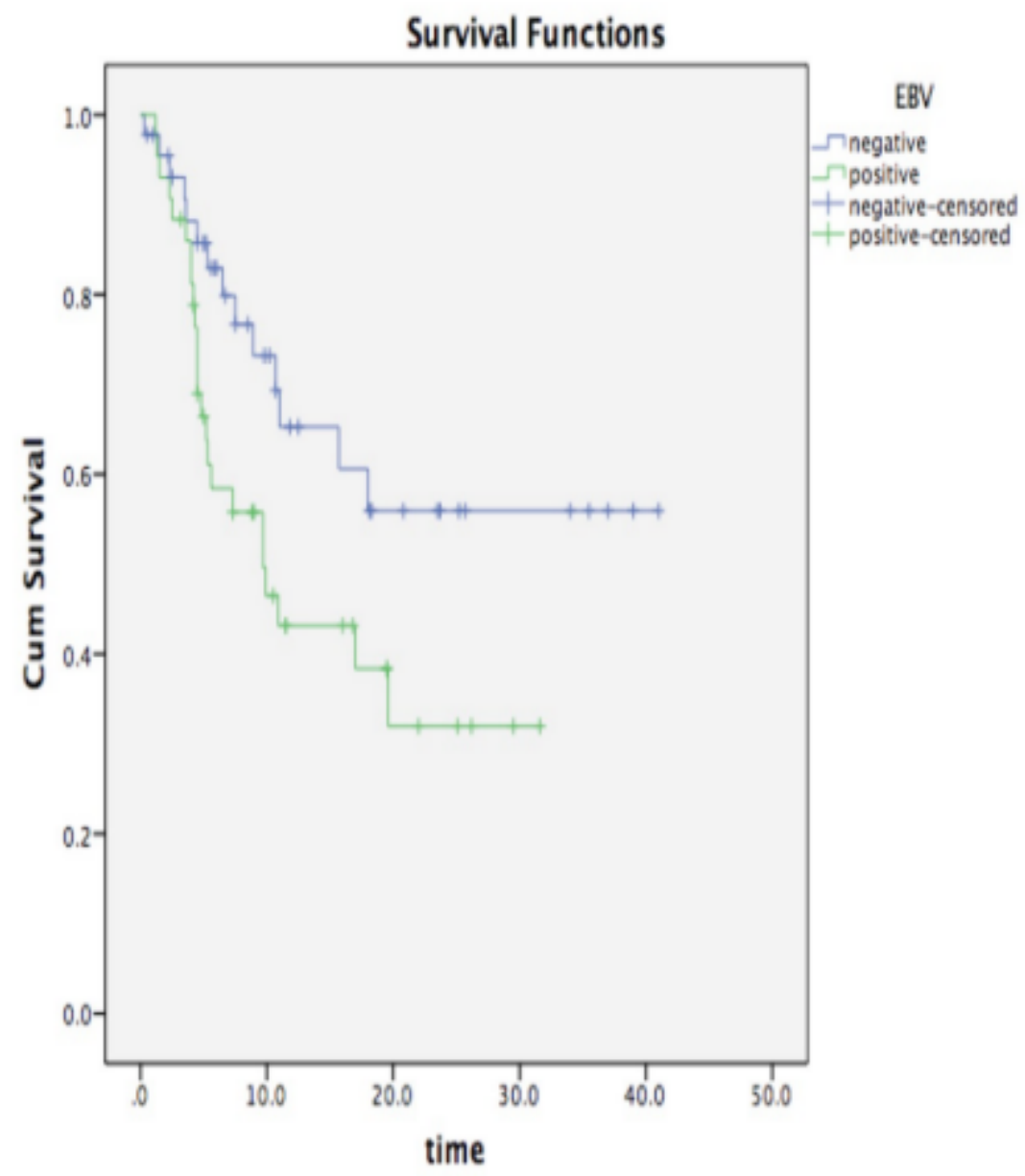

Figure 6

Survival curves of 2 groups whose the first CSF EBV-DNA testing was positive and negative. 


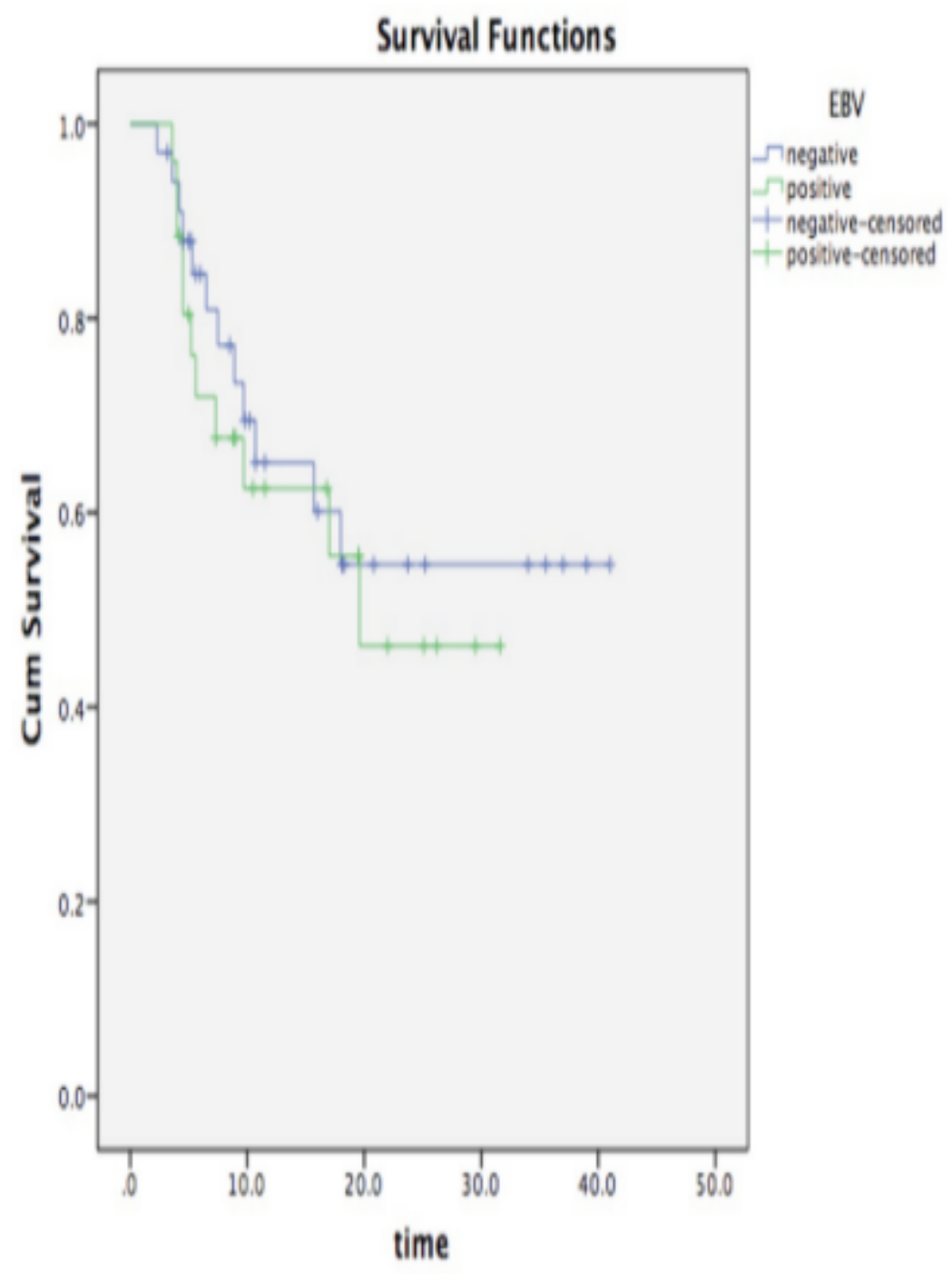

Figure 7

Survival curves of 2 groups whose EBV-DNA testing results before allo-HSCT was positive and negative. 


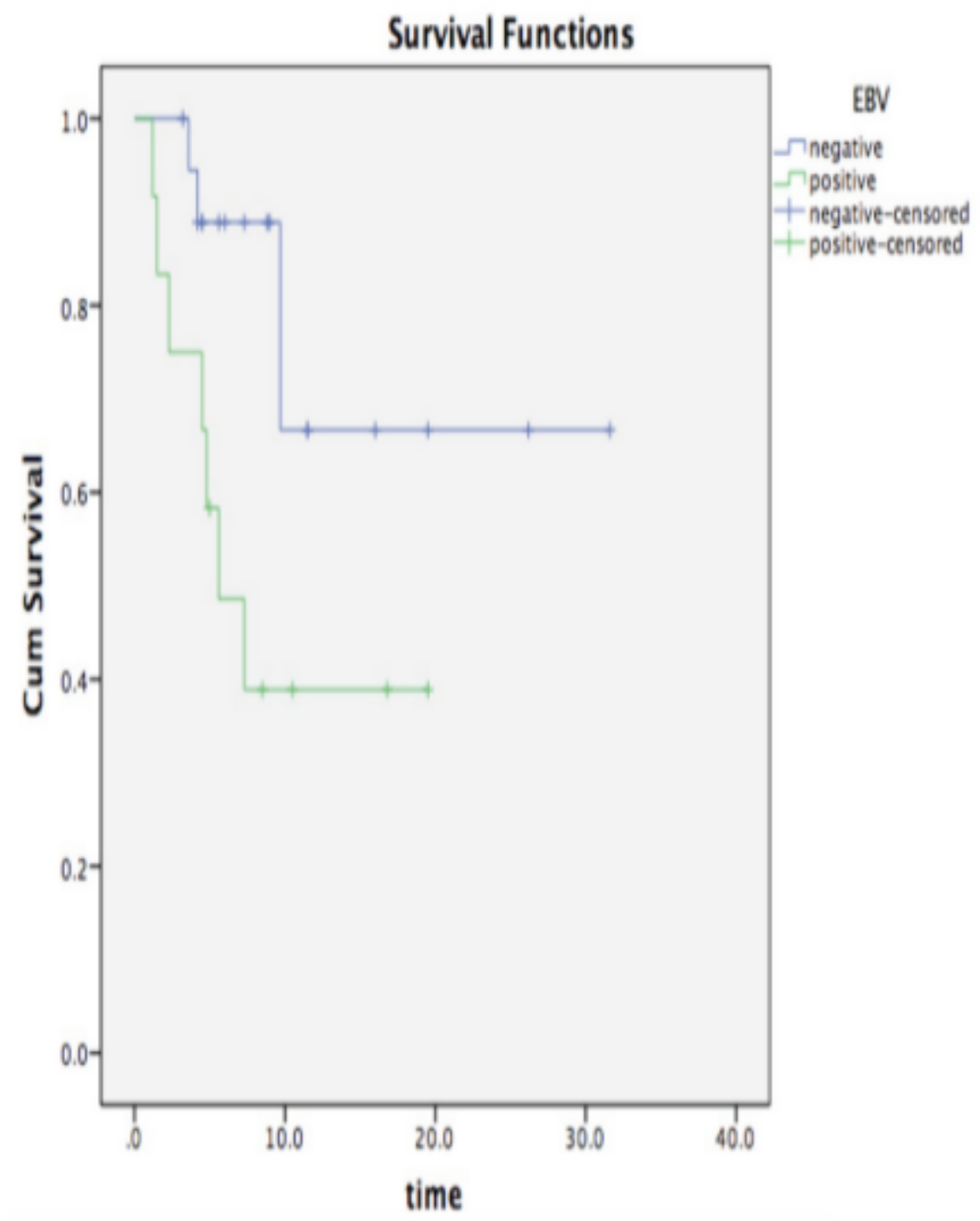

Figure 8

Survival curves of 2 groups whose last CSF EBV-DNA testing was positive and negative. 


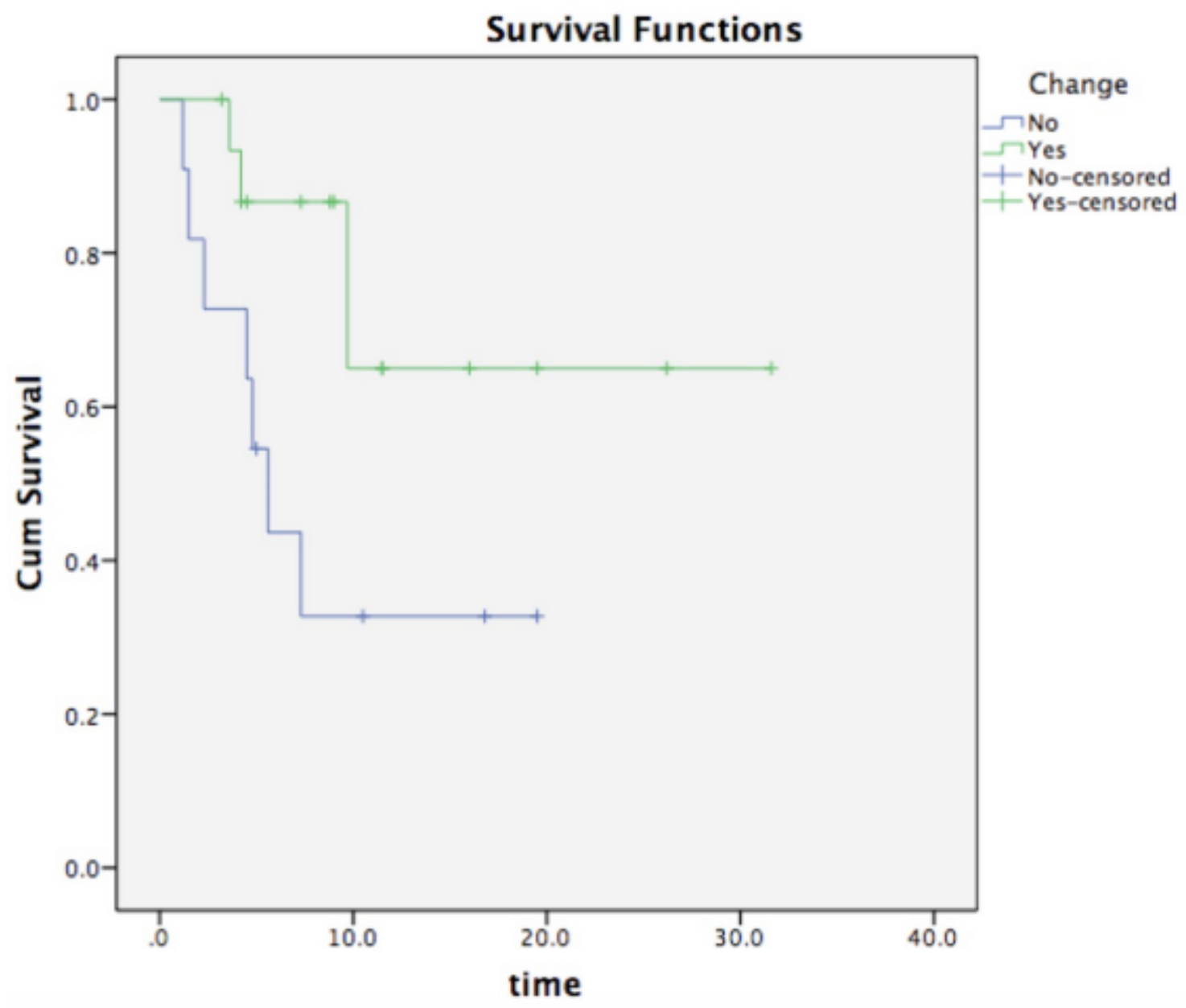

Figure 9

Survival curves of 2 groups whose CSF EBV-DNA testing results whether changed from positive to negative. 


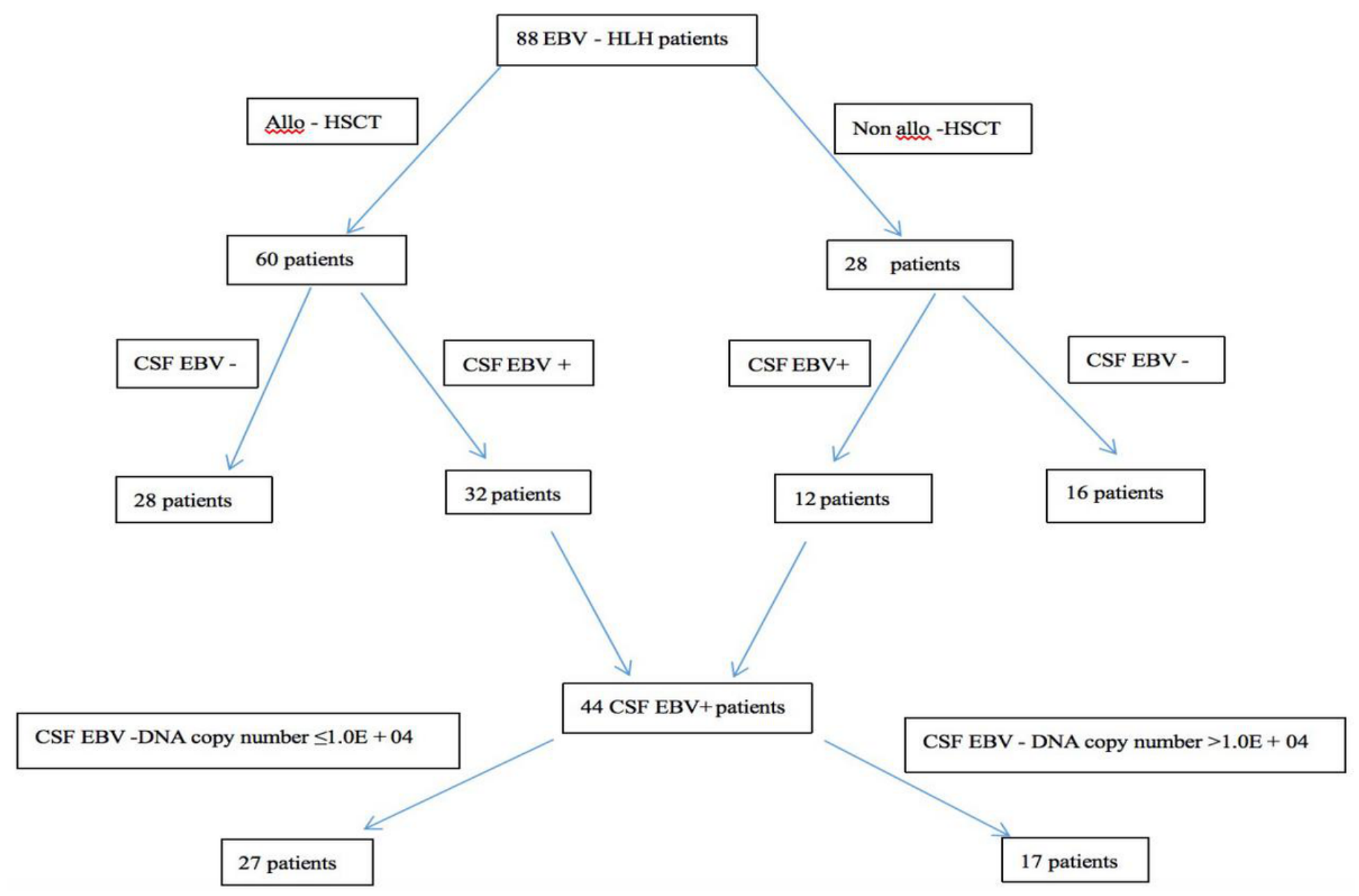

\section{Figure 10}

The flow chart of 88 EBV-HLH patients' information include allo-HSCT and the highest EBV-DNA copy number in CSF. 


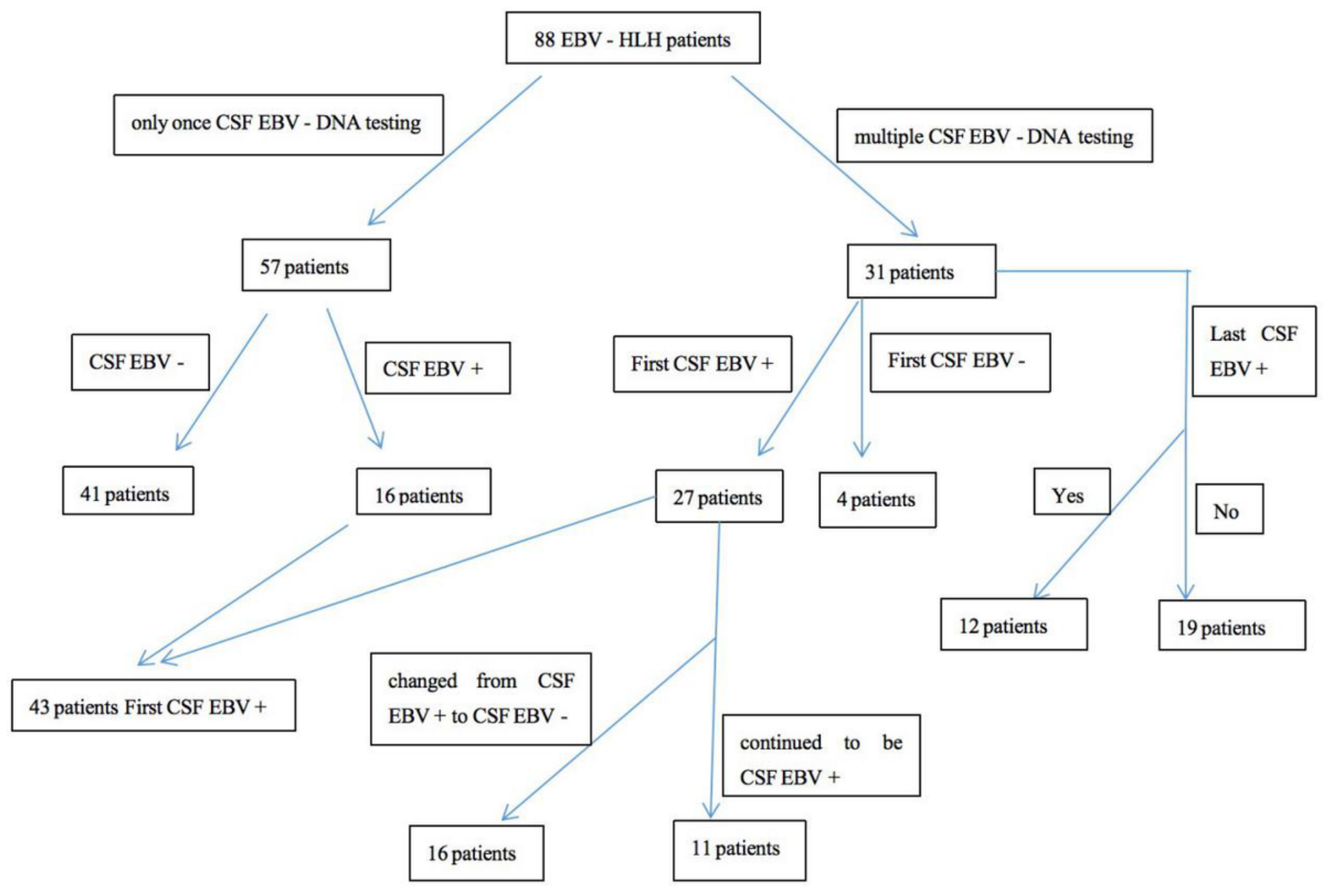

Figure 11

The flow chart of 88 EBV-HLH patients' information of different CSF EBV-DNA testing outcome. 